\title{
ANÁLISE DE UM PROBLEMA DE DIFUSÃO-CONVECÇÃO E REAÇÃO EM SUPERFÍCIE BASEADO EM UM BIOSSENSOR UTILIZANDO O MÉTODO DAS LINHAS (MOL): MODELAGEM MATEMÁTICA COM CAMADA DE HIDROGEL
}

\author{
C.A.M. da SILVA, E.N. MACÊDO e J.N.N. QUARESMA \\ Universidade Federal do Pará, Faculdade de Engenharia Química \\ E-mail para contato: carlos_ms26@yahoo.com.br
}

\begin{abstract}
RESUMO - O Método das Linhas (MOL) foi usado para estudar o processo de transporte de massa e o subjacente processo de interação entre biomoléculas, baseado em uma nova tecnologia de biossensores ópticos. Um modelo matemático foi desenvolvido, a partir da equação de conservação da matéria, acoplada com uma equação cinética que descreve a ligação reversível entre biomoléculas em instrumentos BIACORE. Será considerado um modelo mais real e completo de transferência de massa levando-se em consideração a camada de hidrogel. Foi construído um código computacional baseado em linguagem de programação FORTRAN 90/95, usando-se a subroutina DIVPAG da biblioteca do IMSL, para solucionar numericamente, o sistema de equações diferenciais ordinárias resultantes da aplicação do MOL no modelo proposto. Os resultados numéricos foram gerados e comparados com diferentes metodologias de solução e parâmetros típicos encontrados em ensaios de ligação BIACORE para verificar e validar a modelagem matemática.
\end{abstract}

\section{INTRODUÇÃO}

O estudo das interações moleculares é de fundamental importância para a compreensão do reconhecimento molecular, funções biológicas e fornecem um fundamento químico para todos os processos celulares (MYSZKA, 1997a e SIKAVITSAS et al., 2002). E para estudar essas interações moleculares, uma nova tecnologia de sensores ópticos baseada no fenômeno chamado de ressonância plasmônica de superfície (do inglês surface plasmon resonance - SPR) tem sido cada vez mais utilizada, os biossensores ópticos.

Usando este fenômeno, vários biossensores foram criados no passado com uma variedade de aplicações nos campos da biologia, biomedicina e bioquímica. Entre as várias técnicas disponíveis para analisar as interações biomoleculares, o sistema de tecnologia Biacore é um dos mais largamente utilizados para o monitoramento em tempo real e provou ser um conveniente sistema para uma grande faixa de tamanhos moleculares a partir de pequenos ligantes até células inteiras (GOPINATHA, 2010).

Os biossensores ópticos têm sido usados para caracterizar uma variedade de interações incluindo antígeno-anticorpo, proteínas com oligonucleotídeos ou carboidratos (MYSZKA, 1997b). 
Além destes, o estabilizado método de interação baseado no sistema Biacore pode ser empregado em diversas outras aplicações como seleção de aptâmero, que é um tipo de ácido nucleico capaz de se ligar facilmente a uma molécula-alvo, analise de interações de peptídeos com proteínas, interações de proteínas com proteínas, proteínas com DNA, proteínas com RNA, células com ligantes, hibridização de DNA, proteínas com lipídeos, mapeamento de epítopos, análises cinéticas, processos de seleção de outros parceiros de ligação e dentre outras aplicações (GOPINATHA, 2010). Segundo Myszka (1997b), o instrumento pode investigar ainda, processos de montagem molecular e purificação de proteínas.

Portanto, o uso da tecnologia de biossensores Biacore em diversas áreas de aplicações que vão desde a pesquisa básica em planejamento racional de fármacos, interações biomoleculares e pesquisa biomédica (incluindo câncer, neurobiologia e doenças infecciosas), torna-se necessário a realização de uma modelagem e simulação computacional do processo de transferência de massa com o subjacente processo de interação cinética entre biomoléculas, o que pode favorecer o desenvolvimento de biossensores e compreender melhor os fenômenos envolvidos nesses dispositivos.

\section{FORMULAÇÃO MATEMÁTICA DO PROBLEMA E METODOLOGIA DE SOLUÇÃO}

\subsection{Formulação matemática do problema}

A Figura 1 mostra esquematicamente o biossensor óptico Biacore usado para a simulação experimental:

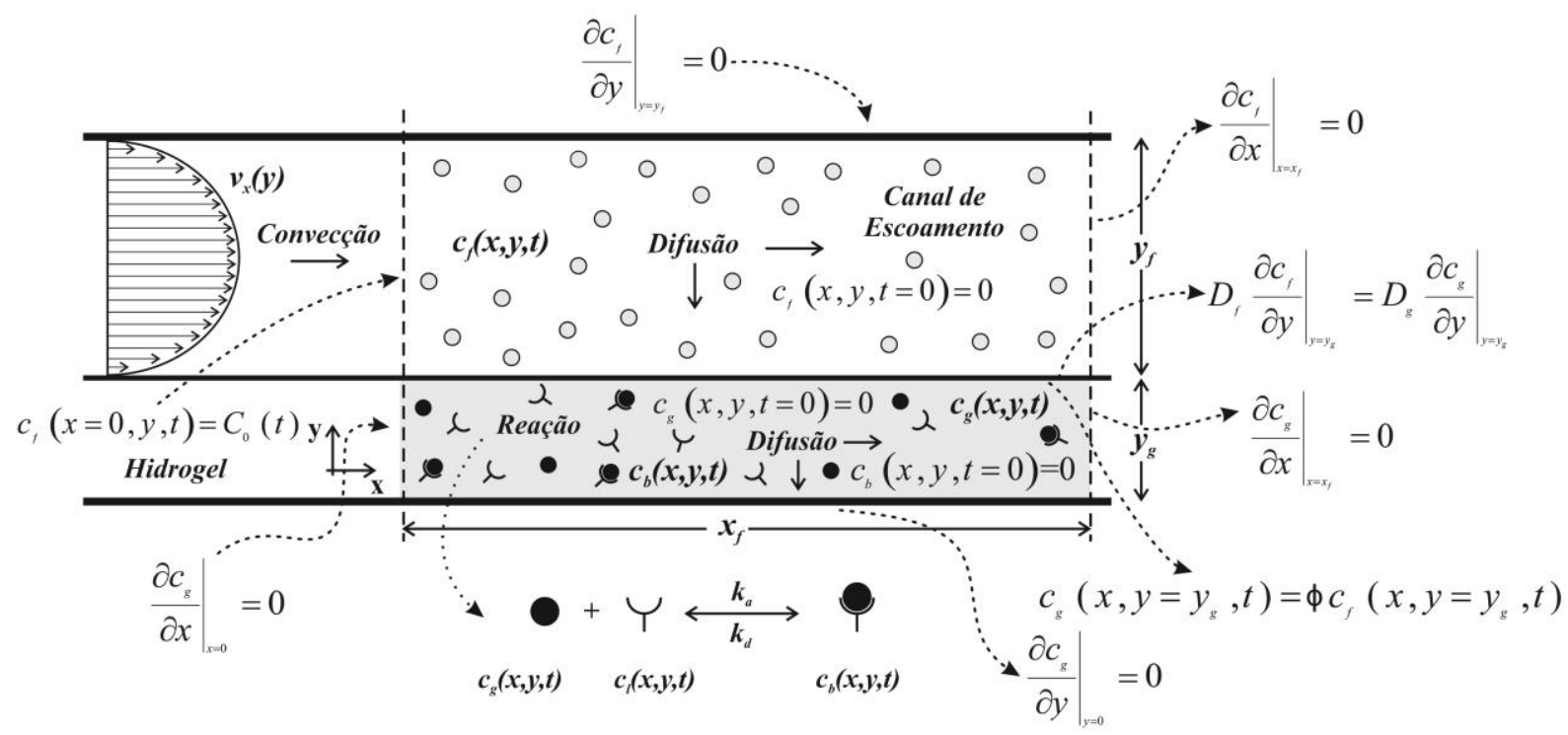

Figura 1 - Visualização esquemática de uma célula de escoamento Biacore com camada de hidrogel. 
O biossensor é composto de duas regiões, o canal de escoamento do analito e a camada do hidrogel. $\mathrm{O}$ escoamento do analito ocorre em um canal de placas paralelas. $\mathrm{O}$ transporte de massa do analito no canal de escoamento ocorre por difusão e convecção sob condições de escoamento laminar completamente desenvolvido. Na camada de hidrogel, a convecção é negligenciada e ocorre a difusão do analito a partir do canal de escoamento para a camada de hidrogel onde os receptores imobilizados encontram-se, ocorrendo a reação de associação e dissociação. A concentração de analito livre no canal de escoamento e na camada de hidrogel, e do complexo analito-receptor na camada de hidrogel são denotadas, respectivamente por $c_{f}(x, y, t), c_{g}(x, y, t)$ e $c_{b}(x, y, t)$. A concentração de receptores livres é $c_{l}(x, y, t)$, onde a concentração total de receptores imobilizados é dada por $C_{T}=c_{l}+c_{b}$. Em razão da largura no canal ser 10 vezes maior do que a altura no canal, considera-se que o gradiente de concentração ao longo da largura do canal seja desprezível.

Propondo-se as seguintes variáveis adimensionais mostradas nas Equações 1.a-r, que foram substituídos no modelo matemático na forma dimensional e a partir das considerações realizadas, temse um novo modelo matemático na forma adimensionalizada mostrado nas Equações 2.a-o:

$$
\begin{aligned}
& X=x / x_{f} ; y_{T}=y_{g}+y_{f} ; Y=y / y_{T} ; \gamma=y_{g} / y_{T} ; \tau=t D / y_{f}^{2} ; C_{f}=c_{f} / C_{0} ; C_{g}=c_{g} / C_{0} ; \\
& C_{b}=c_{b} / C_{T} ; P=6 \bar{v} y_{f} / D_{f} ; \varepsilon_{1}=y_{f} / x_{f} ; \varepsilon_{2}=y_{f} / y_{T} ; \varepsilon_{3}=y_{g} / y_{f} ; \varepsilon_{4}=y_{g} / x_{f} ; \\
& D a=k_{a} C_{T} y_{g}{ }^{2} / D_{f} ; \bar{K}=k_{d} / k_{a} C_{0} ; \overline{\bar{K}}=k_{d} / k_{a} C_{T} ; D=D_{g} / D_{f} ; \bar{C}=C_{0} / C_{T}
\end{aligned}
$$

onde $C_{f}$ e $C_{g}$ são as concentrações adimensionais do analitro livre no canal de escoamento e na camada de hidrogel, respectivamente e $C_{b}$ é a concentração adimensional do complexo analito-receptor na camada de hidrogel. $X$ e $Y$, são as coordenadas axial e transversal adimensionalizada, respectivamente, $\gamma$ a relação entre a altura da camada de hidrogel e a altura total dos dois compartimentos, e $\tau$, o tempo adimensional.

$$
\begin{aligned}
& \frac{\partial C_{f}}{\partial \tau}=\varepsilon_{1}^{2} \frac{\partial^{2} C_{f}}{\partial X^{2}}+\varepsilon_{2}^{2} \frac{\partial^{2} C_{f}}{\partial Y^{2}}-v(Y) \frac{\partial C_{f}}{\partial X} ; 0<X<1 ; \gamma<Y<1 ; \tau>0 \\
& \varepsilon_{3}^{2} \frac{\partial C_{g}}{\partial \tau}=D\left(\varepsilon_{4}^{2} \frac{\partial^{2} C_{g}}{\partial X^{2}}+\gamma^{2} \frac{\partial^{2} C_{g}}{\partial Y^{2}}\right)-D a\left[C_{g}\left(1-C_{b}\right)-\bar{K} C_{b}\right] ; \begin{array}{l}
0<X<1 \\
0<Y<\gamma
\end{array} ; \tau>0 \\
& \varepsilon_{3}^{2} \frac{\partial C_{b}}{\partial \tau}=D a\left[C_{g} \overline{\bar{C}}\left(1-C_{b}\right)-\overline{\bar{K}} C_{b}\right] ; 0<X<1 ; 0<Y<\gamma ; \tau>0 \\
& v(Y)=\varepsilon_{1} P\left(Y\left(1+\varepsilon_{3}\right)-\varepsilon_{3}\right)\left(1-\left(Y\left(1+\varepsilon_{3}\right)-\varepsilon_{3}\right)\right) \\
& C_{f}(X, Y, \tau=0)=0 ; 0<X \leq 1 ; \gamma \leq Y \leq 1
\end{aligned}
$$


$C_{b}(X, Y, \tau=0)=0 ; 0 \leq X \leq 1 ; 0 \leq Y \leq \gamma$

$C_{g}(X, Y, \tau=0)=0 ; 0 \leq X \leq 1 ; 0 \leq Y \leq \gamma$

$C_{f}(X=0, Y, \tau)=C_{0}(\tau) ; C_{0}(\tau)=\left\{\begin{array}{cc}1, & 0 \leq \tau \leq \tau_{i n j} \\ 0, & \tau>\tau_{i n j}\end{array} ; \gamma \leq Y \leq 1\right.$

$\left.\frac{\partial C_{f}}{\partial X}\right|_{X=1}=0 ; \gamma \leq Y \leq 1, \quad \tau>0$

$\left.\frac{\partial C_{f}}{\partial Y}\right|_{Y=1}=0 ; 0 \leq X \leq 1 ; \tau>0$

$\left.\frac{\partial C_{f}}{\partial Y}\right|_{Y=\gamma}=\left.D \frac{\partial C_{g}}{\partial Y}\right|_{Y=\gamma} ; 0 \leq X \leq 1 ; \tau>0$

$\left.\frac{\partial C_{g}}{\partial X}\right|_{X=0}=\left.\frac{\partial C_{g}}{\partial X}\right|_{X=1}=0 ; 0 \leq Y \leq \gamma ; \tau>0$

$\left.\frac{\partial C_{g}}{\partial Y}\right|_{Y=0}=0 ; C_{g}(X, Y=\gamma, \tau)=\phi C_{f}(X, Y=\gamma, \tau) ; 0 \leq X \leq 1 ; \tau>0$

\subsection{Metodologia de Solução - Aplicação do Método das Linhas (MOL)}

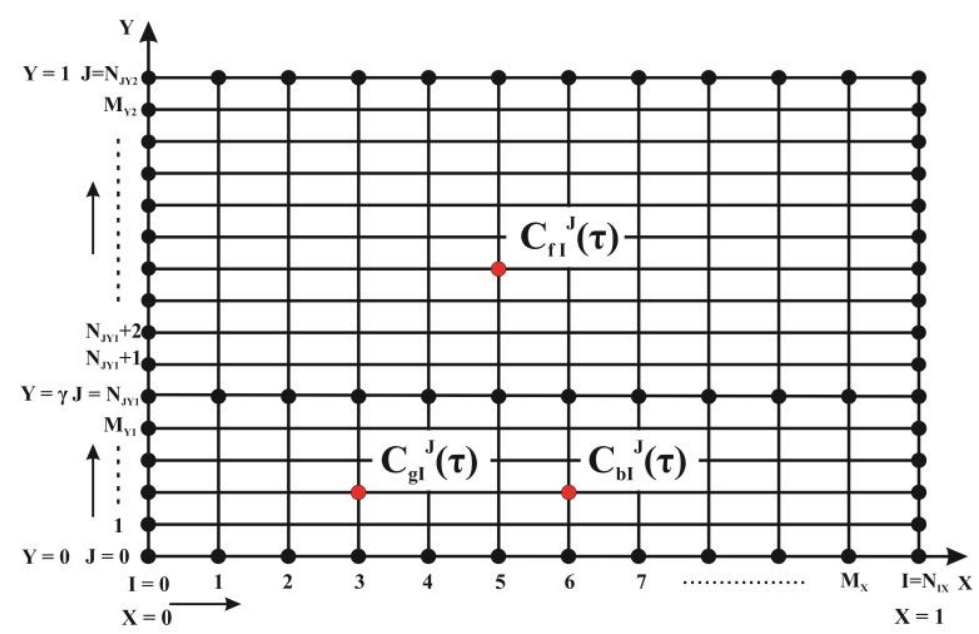

Figura 2 - Esquema de discretização das variáveis espaciais adimensionais. 
Para solucionar o modelo matemático proposto na forma adimensionalizada, será desenvolvido um procedimento baseado no Método das Linhas propondo uma malha uniforme ou regular para as coordenadas espaciais. Através das manipulações analíticas realizadas no sistema de equações diferenciais parciais na forma adimensionalizada por meio de fórmulas de diferenças finitas, pôde-se transformar o sistema de equações diferenciais parciais em um sistema de equações diferenciais ordinárias conforme mostrado na Figura 2. Portanto, o sistema de equações diferenciais ordinárias dependentes no tempo, resultante da aplicação do MOL é dado por:

$$
\begin{aligned}
& \frac{d C_{f I}^{J}}{d \tau}=\varepsilon_{1}^{2} \delta_{f X I}^{J}+\varepsilon_{2}^{2} \delta_{f Y I}^{J}-\Gamma_{X I}^{J} ; 1 \leq I \leq M_{X} ; \mathrm{N}_{J Y 1}+1 \leq J \leq M_{Y 2} \\
& \varepsilon_{3}^{2} \frac{d C_{g I}^{J}}{d \tau}=D\left(\varepsilon_{4}^{2} \delta_{g X I}^{J}+\gamma^{2} \delta_{g Y I}^{J}\right)-D a\left[C_{g I}^{J}\left(1-C_{b I}^{J}\right)-\bar{K} C_{b I}^{J}\right] ; 1 \leq I \leq M_{X} ; 1 \leq J \leq M_{Y 1} \\
& \varepsilon_{3}^{2} \frac{d C_{b I}^{J}}{d \tau}=D a\left[C_{g I}^{J} \overline{\bar{C}}\left(1-C_{b I}^{J}\right)-\overline{\bar{K}} C_{b I}^{J}\right] ; 1 \leq I \leq M_{X} ; 1 \leq J \leq M_{Y 1} \\
& C_{f I}^{J}(\tau=0)=0 ; 0<I \leq N_{I X} ; N_{J Y 1} \leq J \leq N_{J Y 2} \\
& C_{g I}^{J}(\tau=0)=0 ; 0 \leq I \leq N_{I X} ; 0 \leq J \leq N_{J Y 1} \\
& C_{b I}^{J}(\tau=0)=0 ; 0 \leq I \leq N_{I X} ; 0 \leq J \leq N_{J Y 1} \\
& C_{f I=0}^{J}(\tau)=C_{0}(\tau) ; C_{0}(\tau)=\left\{\begin{array}{cc}
1, & 0 \leq \tau \leq \tau_{\text {injecão }} \\
0, & \tau>\tau_{\text {injecão }}
\end{array} ; N_{J Y 1}+1 \leq J \leq N_{J Y}-1\right. \\
& C_{f I=N_{I X}}^{J}(\tau)=\left(4 C_{f I=N_{I X-1}}^{J}(\tau)-C_{f I=N_{I X-2}}^{J}(\tau)\right) / 3 ; N_{J Y 1}+1 \leq J \leq N_{J Y}-1 \\
& C_{f I}^{J=N_{J Y 1}}(\tau)=\left(4 C_{f I}^{J=N_{J Y 1}+1}(\tau)-C_{f I}^{J=N_{J Y 1}+2}(\tau)+\alpha\left(4 C_{g I}^{J=N_{J Y 1}-1}(\tau)-C_{g I}^{J=N_{J Y 1}-2}(\tau)\right)\right) / \\
& (3(1+\phi \alpha)) ; 0 \leq I \leq N_{I X} \\
& C_{f I}^{J=N_{J Y 2}}(\tau)=\left(4 C_{f I}^{N_{J Y 2}-1}(\tau)-C_{f I}^{N_{J Y 2}-2}(\tau)\right) / 3 ; 0 \leq I \leq N_{I X} \\
& C_{g I=0}^{J}(\tau)=\left(4 C_{g I=1}^{J}(\tau)-C_{g I=2}^{J}(\tau)\right) / 3 ; 0 \leq J \leq N_{J Y 1} \\
& C_{g I=N_{I X}}^{J}(\tau)=\left(4 C_{g I=N_{I X-1}}^{J}(\tau)-C_{g I=N_{I X-2}}^{J}(\tau)\right) / 3 ; 0 \leq J \leq N_{J Y 1} \\
& C_{g I}^{J=0}(\tau)=\left(4 C_{g I}^{J=1}(\tau)-C_{g I}^{J=2}(\tau)\right) / 3 ; 0 \leq I \leq N_{I X}
\end{aligned}
$$




$$
C_{g I}^{J=N_{J Y 1}}(\tau)=\phi C_{f I}^{J=N_{J Y 1}}(\tau) ; 0 \leq I \leq N_{I X}
$$

Onde os seguintes termos foram definidos:

$$
\begin{aligned}
& \delta_{f X I}^{J}=\left(C_{f I-1}^{J}(\tau)-2 C_{f I}^{J}(\tau)+C_{f I+1}^{J}(\tau)\right) / \Delta X^{2} \\
& \delta_{f Y I}^{J}=\left(C_{f I}^{J-1}(\tau)-2 C_{f I}^{J}(\tau)+C_{f I}^{J+1}(\tau)\right) / \Delta Y_{2}^{2} \\
& \delta_{g X I}^{J}=\left(C_{g I-1}^{J}(\tau)-2 C_{g I}^{J}(\tau)+C_{g I+1}^{J}(\tau)\right) / \Delta X^{2} \\
& \delta_{g Y I}^{J}=\left(C_{g I}^{J-1}(\tau)-2 C_{g I}^{J}(\tau)+C_{g I}^{J+1}(\tau)\right) / \Delta Y_{1}^{2} \\
& \Gamma_{X I}^{J}=\left\{\begin{array}{l}
\mathrm{U}_{\mathrm{J}}\left(C_{f I}^{J}(\tau)-C_{f I-1}^{J}(\tau)\right) / \Delta X \quad \therefore \mathrm{U}_{\mathrm{J}}>0 \\
\mathrm{U}_{\mathrm{J}}\left(C_{f I+1}^{J}(\tau)-C_{f I}^{J}(\tau)\right) / \Delta X \quad \therefore \mathrm{U}_{\mathrm{J}}<0
\end{array}\right. \\
& \alpha=D \Delta Y_{2} / \Delta Y_{1} ; \mathrm{U}_{\mathrm{J}}=\varepsilon_{1} P\left(Y\left(1+\varepsilon_{3}\right)-\varepsilon_{3}\right)\left(1-\left(Y\left(1+\varepsilon_{3}\right)-\varepsilon_{3}\right)\right)
\end{aligned}
$$

O conjunto de Equações 3.a-s forma um sistema finito de equações diferenciais ordinárias acopladas na variável temporal adimensional que deve ser truncado em NIX e NJY1=NJY2 intervalos suficiente para computar os campos $\mathrm{Cf}(\mathrm{X}, \mathrm{Y}, \tau), \mathrm{Cg}(\mathrm{X}, \mathrm{Y}, \tau)$ e $\mathrm{Cb}(\mathrm{X}, \mathrm{Y}, \tau)$, dentro de uma precisão prescrita até atingir uma convergência dos resultados numéricos obtidos. Para a solução de tal sistema, será desenvolvido um código computacional em linguagem de programação FORTRAN 90/95, utilizando-se a subroutina DIVPAG, especializada em problemas de valor inicial, a partir da biblioteca do IMSL.

\section{RESULTADOS E DISCUSSÃO}

Os parâmetros experimentais utilizados para a simulação experimental do processo de interação biomolecular em uma célula de escoamento da Figura 1 foram retirados de Lebedev et al. (2006), o qual resolveu numericamente o modelo matemático através de um pacote computacional chamado Femlab 3.0. Os parâmetros utilizadas na simulação representam casos típicos usados em experimentos de biossensores Biacore e são mostrados na Tabela 1.

Depois de realizado uma análise de convergência (não mostrado aqui), verificou-se uma convergência numéricos dos resultados com 4 algarismos significativos com números de intervalos $N_{I X}=N_{J Y I}=N_{J Y 2}=20$. Portanto, com 20 intervalos consegue-se descrever de forma satisfatória as concentrações do analito livre no canal de escoamento e na camada de hidrogel e também a concentração do complexo analito-receptor na camada de hidrogel. 
Tabela 1 - Parâmetros experimentais de simulação para Lebedev et al. (2006).

\begin{tabular}{|c|c|c|c|c|c|}
\hline \multicolumn{6}{|c|}{ Canal de Escoamento } \\
\hline $\begin{array}{r}x_{f}=10^{-3} \mathrm{~m} \\
y_{f}=5 \times 10^{-5}\end{array}$ & $k_{M}=1,28 \times 10^{-5} \mathrm{~m} . \mathrm{s}$ & \multicolumn{2}{|c|}{$D_{f}=10^{-10} \mathrm{~m}^{2} \cdot \mathrm{s}^{-1}$} & $\begin{array}{c}v_{m}=5 \times 10^{-3} \mathrm{~m} . \mathrm{s} \\
P=10000\end{array}$ & $C_{0}=10^{-5} \mathrm{~mol} . \mathrm{m}$ \\
\hline \multicolumn{6}{|c|}{ Camada de Hidrogel } \\
\hline \multicolumn{3}{|c|}{$C_{T}=10^{-1}$ mol. $\mathrm{m}^{-3}$} & \multicolumn{2}{|c|}{$D_{g}=0,05 D_{f}$} & $\phi=0,1$ \\
\hline \multicolumn{3}{|c|}{ Caso A } & \multicolumn{3}{|c|}{ Caso B } \\
\hline \multicolumn{3}{|c|}{$\begin{array}{l}k_{a}=4 \times 10^{2} \mathrm{~m}^{3} \cdot \mathrm{mol}^{-1} \cdot \mathrm{s}^{-1}, k_{d}=10^{-3} \mathrm{~s}^{-1} \\
k_{a} C_{T y_{g}}=4,0 \times 10^{-6} \mathrm{~m} \cdot \mathrm{s}^{-1}, \mathrm{Da}=0,004\end{array}$} & \multicolumn{3}{|c|}{$\begin{array}{l}k_{a}=4 \times 10^{3} m^{3} \cdot m o l^{-1} \cdot s^{-1}, k_{d}=10^{-2} \mathrm{~s}^{-1} \\
k_{a} C_{T} y_{g}=4,0 \times 10^{-5} \mathrm{~m} \cdot \mathrm{s}^{-1}, D a=0,04\end{array}$} \\
\hline
\end{tabular}

Nas Figuras 3.a-b, são mostrados a comparação dos resultados obtidos neste presente trabalho e pelos resultados desenvolvidos por Lebedev et al. (2006).
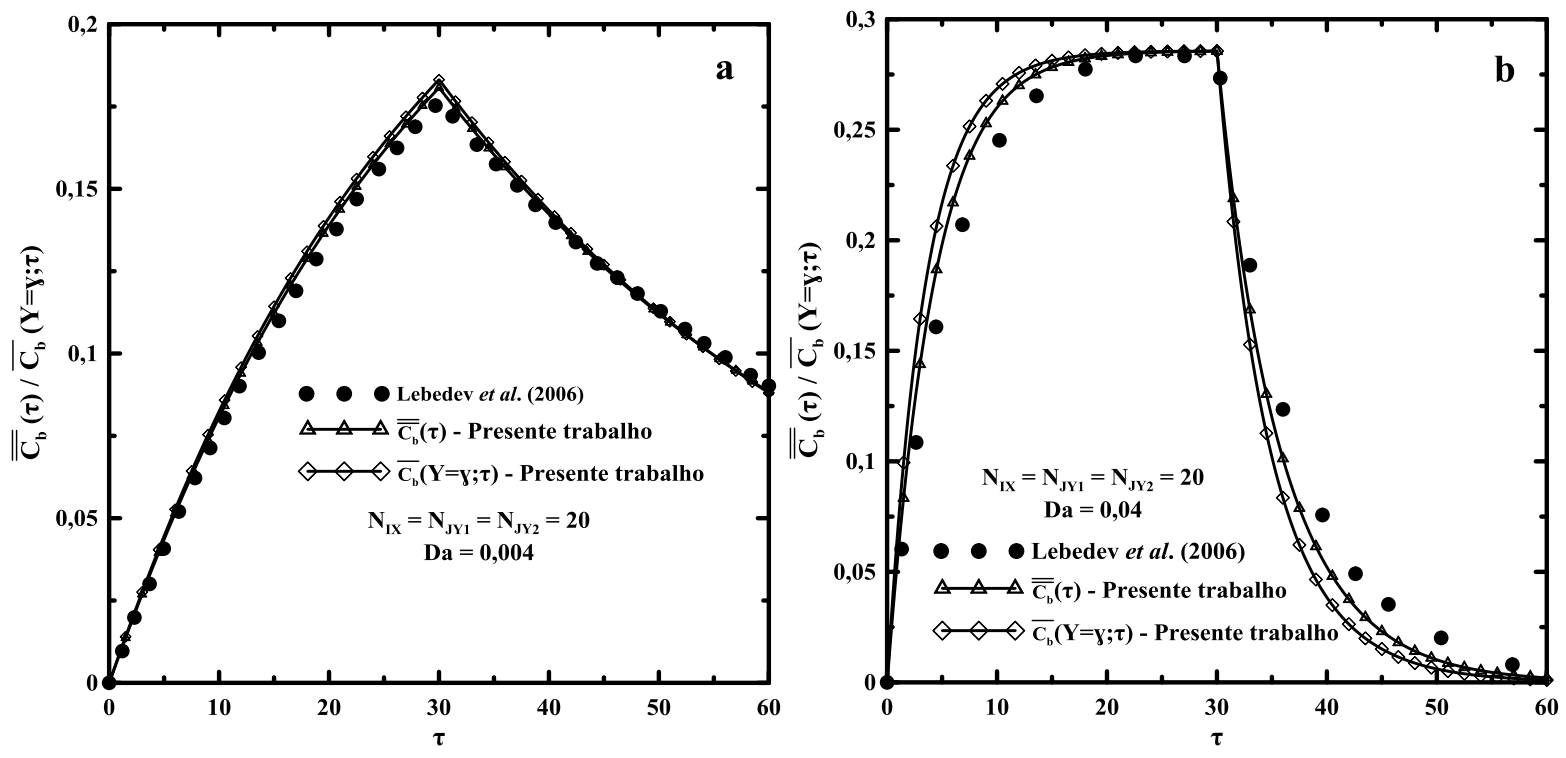

Figura 3 - Comparação dos resultados obtidos pelo presente trabalho (MOL) com o desenvolvido por Lebedev et al.(2006) para (a) $\mathrm{Da}=0,004$ e (b) $\mathrm{Da}=0,04$.

Os resultados gerados pelo presente trabalho mostram uma boa concordância com os resultados obtidos por Lebedev et al. (2006). As Figuras 3.a-b, mostram a concentração média do complexo analito-receptor na forma adimensional ao longo do tempo para dois diferentes valores de Da, onde para $D a=0,004$, não existem limitações do transporte de massa e sim da reação de ligação entre o analito e o receptor, e para $D a=0,04$, as limitações do transporte de massa estão presentes de forma reduzida. Os resultados para ambas as concentrações médias do complexo analito-receptor são praticamente os mesmos. Isto pode acontecer devido ao fato que a espessura da camada de hidrogel ser muito pequena, o analito se distribui mais rapidamente dentro na camada de hidrogel e também pelo fato de que apesar do livre caminho médio ser maior que a espessura da camada de hidrogel, sua 


\section{9 a 22 de outubro de 2014 \\ Florianópolis/SC}

ordem de magnitude não é tão elevada assim, de modo que pode-se considerar, de acordo com os parâmetros experimentais de simulação, que os receptores estão imobilizados diretamente sobre a superfície do sensor.

\section{CONCLUSÕES}

No presente trabalho, foi desenvolvido um estudo teórico sobre o processo de transporte de massa e o subjacente processo de ligação de biomoléculas em superfície baseado em um biossensor Biacore. Realizou-se uma modelagem matemática mais real e completa baseado em um problema de difusão-convecção-reação, com a aplicação do Método das Linhas (MOL) para resolver o sistema acoplado de equações diferenciais parciais que descrevem o problema físico. Enfoque maior foi dado na modelagem físico-química do que em analisar as aplicações práticas particulares, e extensões do modelo podem ser facilmente realizadas para estudar outros fenômenos envolvidos em células de escoamento de biossensores. Os resultados apresentados neste trabalho foram testados com outra metodologia de solução disponíveis na literatura, mostrando-se coerente e fisicamente consistente com os parâmetros experimentais e metodologias reportadas na literatura. A abordagem aplicada neste trabalho foi adequada para predizer a concentração média do complexo analito-receptor, para as condições analisadas e mostram-se em boa concordância com os resultados obtidos na literatura. $\mathrm{O}$ presente trabalho contribui cientificamente para o desenvolvimento de biossensores ópticos. Devido a várias áreas tecnológicas de aplicação e uso dos mesmos, como por exemplo, o uso de biossensores na detecção precoce e tratamento de câncer, podendo ser importantes ferramentas para usos na medicina, desenvolvimentos de fármacos e tantas outras aplicações. Portanto, o uso dos modelos matemáticos para descrever todos os fenômenos envolvidos no processo é de fundamental importância para conseguir esses grandes avanços tecnológicos.

\section{REFERÊNCIAS}

MYSZKA, D. G. Kinetic analysis of macromolecular interactions using surface plasmon resonance biosensors. Journal of Biotechnology, v. 8, p. 50-57, 1997a.

MYSZKA, D. G.; MORTON, T. A.; DOYLE, M. L.; CHAIKEN, I. M. Kinetic analysis of a protein antigen-antibody interaction limited by mass transport on an optical biosensor. Journal of Biophysical Chemistry, v. 64, p.127-137, $1997 \mathrm{~b}$.

SIKAVITSAS, V.; NITSCHE, J. M.; MOUNTZIARIS, T. J. Transport and Kinetic Processes Underlying Biomolecular Interactions in the BIACORE Optical Biosensor. Journal of Biotechnology Progress, v.18, p.885-897. 2002.

GOPINATHA, S. C. B. Biosensing applications of surface plasmon resonance-based Biacore technology. Journal of Sensors and Actuators B, v. 150, p. 722-733, 2010.

LEBEDEV, K.; MAFÉ, S.; STROEVE, P. Convection, diffusion and reaction in a surface-based biosensor: Modeling of cooperativity and binding site competition on the surface and in the hydrogel. Journal of Colloid and Interface Science, v.296, p.527-537, 2006. 